\title{
Implementation of a Scenario-based MPC for HVAC Systems: an Experimental Case Study *
}

\author{
Alessandra Parisio* Damiano Varagnolo** Marco Molinari* \\ Giorgio Pattarello* Luca Fabietti ${ }^{* * *}$ Karl H. Johansson* \\ * School of Electrical Engineering, Royal Institute of Technology, \\ Osquldas väg 10, Stockholm, Sweden \\ ** Department of Computer Science, Electrical and Space Engineering, \\ Lulea University of Technology, Forskargatan 1, Sweden \\ *** Department of Information Engineering, University of Padova, via \\ Gradenigo 6/b, Italy
}

\begin{abstract}
Heating, Ventilation and Air Conditioning (HVAC) systems play a fundamental role in maintaining acceptable thermal comfort and air quality levels. Model Predictive Control (MPC) techniques are known to bring significant energy savings potential. Developing effective MPC-based control strategies for HVAC systems is nontrivial since buildings dynamics are nonlinear and influenced by various uncertainties. This complicates the use of MPC techniques in practice. We propose to address this issue by designing a stochastic MPC strategy that dynamically learns the statistics of the building occupancy patterns and weather conditions. The main advantage of this method is the absence of a-priori assumptions on the distributions of the uncertain variables, and that it can be applied to any type of building. We investigate the practical implementation of the proposed MPC controller on a student laboratory, showing its effectiveness and computational tractability.
\end{abstract}

Keywords: Control applications, Implementation, Model-based and predictive control, Probabilistic models, Control-oriented models, Stochastic control

\section{INTRODUCTION}

Heating, cooling and air conditioning is a necessity in buildings, which account for a major share of the global energy consumption. Reports indicate that HVAC systems in developed countries contribute for approximately one fifth of the total national energy usages (European Commission, 2008). Current practice shows its limits, with potential energy savings achievable by using systematic building management being estimated from $5 \%$ to $30 \%$ of the total consumptions (Costa et al., 2013; Chua et al., 2013).

Literature Review. HVAC control systems performance can be improved by using predictive strategies, like in Goyal et al. (2012); Gwerder and Toedtli (2005); Salsbury et al. (2012); Hua and Karavab (2014). MPC schemes are expected to become a common solution for smart buildings in a few years (Aswani et al., 2012).

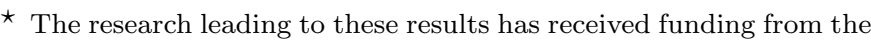
European Union Seventh Framework Programme [FP7/2007-2013] under grant agreement $n^{\circ} 257462$ HYCON2 Network of excellence, the European Institute of Technology (EIT) Information and Communication Technology (ICT) Labs, the Swedish Energy Agency, the Swedish Governmental Agency for Innovation Systems (VINNOVA), the Swedish Research Council and the Knut and Alice Wallenberg Foundation.

Corresponding author: Alessandra Parisio, parisio@kth.se
}

This tendency is supported not only by simulations (Treado and Chen, 2013; Wallace et al., 2012; Fadzli Haniff et al., 2013), but also by some experimental results on real buildings (Sturzenegger et al., 2013; Široký et al., 2011; Parisio et al., 2013b).

Successful implementations will be likely based on stochastic MPC schemes with probabilistic constraints: indoor air conditions are intrinsically affected by stochastic disturbances, such as occupancy patterns and outdoor temperature. Current standards state that the probability of comfort violations should do not exceed certain levels (BSI, 2008).

There is already a vast literature on stochastic MPC schemes for HVAC control. For example, Mady et al. (2011); Ma and Borrelli (2012); Ma et al. (2012); Oldewurtel et al. (2012).

All the previously mentioned approaches restrict disturbances to have Gaussian distribution, assumption that makes the problems solvable. Instead, we proposed a scenario-based tractable approximation of the chance constrained MPC problem, where the scenarios are i.i.d. samples extracted from general probability distributions, thus not restricted to be Gaussian (Parisio et al., 2013a,b). Another scenario-based approach has been proposed by Zhang et al. (2013). Here authors propose an iterative bilinearization of the building model around nominal 
trajectories and sample occupancy scenarios from a set of measurement data collected in eight single offices equipped with motion sensors. The numerical simulations performed in this work suggest that scenarios-based techniques outperform other predictive methods.

Statement of contributions. With respect to the state-ofthe-art literature, we: i) propose a novel building model, which better captures the building dynamics while maintaining linearity assumptions; ii) develop and implement on a real testbed (the KTH HVAC testbed) an advanced control scheme that continuously adapt the operation of the HVAC system to unknown disturbances while guaranteeing occupants comfort and wellbeing. More precisely, the new model accounts for minimal ventilation levels and more precise actuators dynamics. We then compare and analyze the performance 3 control schemes applied to the KTH HVAC testbed. The first controller is the current practice in our building. The second is a deterministic MPC disregarding information on the uncertainties of the disturbances. The third controller is instead our novel Scenario-based Model Predictive Control (SMPC) scheme. Results show that the SMPC scheme leads to a more robust and potentially energy efficient behavior of the system.

Structure of the manuscript Section 2 presents the novel building model and related HVAC MPC scheme. Section 3 describes our experimental campaign and Section 4 ends the manuscript with a summary of our conclusions and with indications of the next steps.

\section{SCENARIO-BASED MPC FOR HVAC SYSTEMS}

In this section we first describe the model of the building (Section (2.1)), and then we outline the general structure of the Scenario-based Model Predictive Control (SMPC) control scheme (Section (2.2)).

We remark that, since the overall building energy usage is commonly computed as the sum of the energy usages of the single thermal zones (Gwerder and Toedtli, 2005), here we focus on the control of a single thermal zone (or room).

\subsection{Modeling}

To improve the computational tractability of the overall control problem, we take advantage from the independence of the $\mathrm{CO}_{2}$ concentration dynamics from the thermal ones, which allow us to address two separated subproblems: $i$ ) the $\mathrm{CO}_{2}$-SMPC problem, which aims at minimizing energy use while keeping $\mathrm{CO}_{2}$ levels in given comfort bounds; ii) the T-SMPC problem, controlling instead the indoor temperature.

Here we describe the two separated models for the dynamics under consideration.

Model for the $\mathrm{CO}_{2}$ concentration dynamics The model is derived from a $\mathrm{CO}_{2}$ balance equation accounting for the fresh air from the ventilation system and the amount of $\mathrm{CO}_{2}$ generated per occupant. The state of the model and its output, indicated respectively with $x_{\mathrm{CO}_{2}}$ and $y_{\mathrm{CO}_{2}}$, are set to be equal to $\Delta \mathrm{CO}_{2}$, the nonnegative difference between the $\mathrm{CO}_{2}$ concentration in the room and the inlet air $\mathrm{CO}_{2}$ concentration (the latter assumed equal to outdoor $\mathrm{CO}_{2}$ concentration levels).

The model disturbance $w_{\mathrm{CO}_{2}}$ represents the number of occupants, while the control input is the rate of the air flow coming from the ventilation system, which is denoted by $\dot{m}_{\text {venting. }}^{\mathrm{CO}_{2}}$. This input allows to control the heat flow due to the ventilation system, indicated with $Q_{\text {venting }}$.

The reduction in the indoor $\mathrm{CO}_{2}$ concentration levels induced by $\dot{m}_{\text {venting }}^{\mathrm{CO}_{2}}$ is modeled with the bilinear term $\dot{m}_{\text {venting }}^{\mathrm{CO}_{2}} \cdot x_{\mathrm{CO}_{2}}$. Since linear problems can be solved more efficiently than nonlinear ones, we derive an equivalent linear model of the $\mathrm{CO}_{2}$ concentration dynamics by introducing the auxiliary input $u_{\mathrm{CO}_{2}}:=\dot{m}_{\text {venting }}^{\mathrm{CO}_{2}} \cdot x_{\mathrm{CO}_{2}}$, which then hides the bilinear term defined above. To meet the physical bounds on the original control input $\dot{m}_{\text {venting }}^{\mathrm{CO}_{2}}, u_{\mathrm{CO}_{2}}$ has to satisfy

$$
\dot{m}_{\text {venting }}^{\min } \cdot x_{\mathrm{CO}_{2}} \leq u_{\mathrm{CO}_{2}} \leq \dot{m}_{\text {venting }}^{\max } \cdot x_{\mathrm{CO}_{2}} .
$$

Then, we can then easily derive $\dot{m}_{\text {venting }}^{\mathrm{CO}_{2}}$ by inverting the definition of $u_{\mathrm{CO}_{2}}$.

With the control input $u_{\mathrm{CO}_{2}}$, the $\mathrm{CO}_{2}$ concentration dynamics can eventually be described by the discrete-time Linear Time Invariant (LTI) system

$$
\begin{aligned}
x_{\mathrm{CO}_{2}}(k+1) & =a x_{\mathrm{CO}_{2}}(k)+b u_{\mathrm{CO}_{2}}(k)+e w_{\mathrm{CO}_{2}}(k) \\
y_{\mathrm{CO}_{2}}(k) & =x_{\mathrm{CO}_{2}}(k) .
\end{aligned}
$$

We assume bounds on the input $u_{\mathrm{CO}_{2}}(k)$ of the form $u_{\mathrm{CO}_{2}}^{\min } \leq u_{\mathrm{CO}_{2}}(k) \leq u_{\mathrm{CO}_{2}}^{\max }$, which can be expressed as polytopic constraints $F u_{\mathrm{CO}_{2}}(k) \leq f$. We further define comfort constraints on the indoor $\mathrm{CO}_{2}$ concentration as $0 \leq y_{\mathrm{CO}_{2}}(k) \leq y_{\mathrm{CO}_{2}}^{\max }$. Considering that $x_{\mathrm{CO}_{2}}=y_{\mathrm{CO}_{2}}$, comfort constraints and constraints (1) can be written in a compact form as mixed constraints on the input and on the output, $V_{y} y_{\mathrm{CO}_{2}}(k)+V_{u} u_{\mathrm{CO}_{2}}(k) \leq v$. We refer the reader to Parisio et al. (2013b) for details on the construction of the constraints matrices.

Model for the thermal dynamics We consider a thermal Resistive-Capacitive (RC) network of first-order systems, where the nodes are the states representing the temperatures of the room, walls, floor and ceiling. Each state is associated to a heat transfer differential equation.

The model disturbances represent the outdoor temperature, radiation, internal gains, heat flows due to occupancy, equipments and lightings. The control inputs are the temperature of the supplied air, $T_{\mathrm{sa}}$, the mean radiant temperature of the radiators, $T_{\mathrm{mr}}$, and the air flow rate $\dot{m}_{\text {venting. }}$ (We remind that $\dot{m}_{\text {venting }}$ must be at least equal to $\dot{m}_{\text {venting }}^{\mathrm{CO}_{2}}$, the latter representing the minimum air flow rate needed to maintain optimal $\mathrm{CO}_{2}$ levels.) The inputs $T_{\mathrm{sa}}, T_{\mathrm{mr}}$ and $\dot{m}_{\text {venting }}$ allow to control two different heat flows: i) $Q_{\text {venting, }}$, representing the contribute due to the ventilation system; ii) $Q_{\text {heating }}$, representing the contribute due to the radiators.

We now aim to: $i$ ) hide the bilinear term of the indoor thermal dynamics $Q_{\text {venting }}=\dot{m}_{\text {venting }} c_{\text {pa }}\left(T_{\text {sa }}-T_{\text {room }}\right)$, 
ii) model the contribute due to the requirements on the $\mathrm{CO}_{2}$ concentration levels (i.e., due to the minimal air flow $\dot{m}_{\text {venting }}^{\mathrm{CO}_{2}}$ ) and the absolute value of $Q_{\text {venting }}$ (which is part of the cost function to be minimized).

To achieve these aims, we model the two heat flows as

$$
\begin{aligned}
& Q_{\text {venting }}=\dot{m}_{\text {venting }}^{\mathrm{CO}_{2}} c_{\mathrm{pa}}\left(\Delta T_{h}-\Delta T_{c}\right)+c_{\mathrm{pa}}\left(\Delta u_{h}-\Delta u_{c}\right) \\
& Q_{\text {heating }}=A_{\text {rad }} h_{\mathrm{rad}} \Delta T_{\mathrm{h}, \mathrm{rad}}
\end{aligned}
$$

where $c_{\mathrm{pa}}$ is the specific heat of the dry air, $A_{\mathrm{rad}}$ is the emission area of the radiators, $h_{\text {rad }}$ is the heat transfer coefficient of the radiators, and the nonnegative variables $\Delta T_{h}, \Delta T_{c}, \Delta u_{h}$ and $\Delta u_{c}$ are s.t.

$$
\begin{aligned}
& \Delta T_{h}-\Delta T_{c}=T_{\mathrm{sa}}-T_{\text {room }} \\
& \Delta T_{h}+\Delta T_{c}=\left|T_{\mathrm{sa}}-T_{\text {room }}\right| \\
& \Delta u_{h}-\Delta u_{c}=\Delta \dot{m}_{\text {venting }}\left(T_{\mathrm{sa}}-T_{\text {room }}\right) \\
& \Delta u_{h}+\Delta u_{c}=\Delta \dot{m}_{\text {venting }}\left|T_{\mathrm{sa}}-T_{\text {room }}\right|
\end{aligned}
$$

with $\Delta \dot{m}_{\text {venting }}:=\dot{m}_{\text {venting }}-\dot{m}_{\text {venting }}^{\mathrm{CO}_{2}}$ the additional air flow rate required for guaranteeing the thermal comfort, and $T_{\text {room }}$ the indoor temperature.

With the newly introduced variables, the dynamics of the indoor temperature can be modeled with the discrete-time linear system

$$
\begin{aligned}
x_{\mathrm{T}}(k+1) & =A_{\mathrm{T}} x_{\mathrm{T}}(k)+B_{\mathrm{T}}(k) u_{\mathrm{T}}(k)+E_{\mathrm{T}} w_{\mathrm{T}}(k) \\
y_{\mathrm{T}}(k) & =C_{\mathrm{T}} x_{\mathrm{T}}(k),
\end{aligned}
$$

where the state $x_{\mathrm{T}}(k)$ contains the temperatures of the room and of the inner and outer parts of the walls, $u_{\mathrm{T}}(k):=\left[\Delta T_{h}(k), \Delta T_{c}(k), \Delta u_{h}(k), \Delta u_{c}(k), \Delta T_{h, \operatorname{rad}}(k)\right]$ is the input vector, and $w_{\mathrm{T}}(k)$ is the vector of random disturbances (outdoor temperature, solar radiation and internal heat gains). The output $y_{\mathrm{T}}(k)$ is the indoor temperature at time $k$. We notice that the input matrix $B_{\mathrm{T}}(k)$ is time varying since it depends on $\dot{m}_{\text {venting }}^{\mathrm{CO}_{2}}(k)$.

We represent physical bounds on the original control inputs as

$$
\begin{aligned}
& T_{\mathrm{sa}}^{\min }-T_{\text {room }}(k) \leq \Delta T_{h}(k)-\Delta T_{c}(k) \leq T_{\mathrm{sa}}^{\max }-T_{\text {room }}(k) \\
& \left|\Delta u_{h}(k)-\Delta u_{c}(k)\right| \leq \Delta \dot{m}_{\text {venting }}^{\max }(k)\left|\Delta T_{h}(k)-\Delta T_{c}(k)\right|
\end{aligned}
$$

$$
\text { where } \Delta \dot{m}_{\text {venting }}^{\max }(k):=\dot{m}_{\text {venting }}^{\max }-\dot{m}_{\text {venting }}^{\mathrm{CO}_{2}}(k) \text {. }
$$

Compared to our previous contributions (Parisio et al. 2013a,b), the building model now encompasses a more detailed solar radiation model. Furthermore, the temperature variation in adjacent rooms has been estimated by means of a sinusoidal dependence in time, which proved to be in sufficiently good accordance with measured data.

As outlined above, hard constraints on inputs and constraints (5) can be written in compact form as polytopic constraints on inputs, $F u_{\mathrm{T}}(k) \leq f$. Comfort constraints on the output and constraints (4) can be written in a compact form as mixed constraints on the input and on the output, $V_{y} y_{\mathrm{T}}(k)+V_{u} u_{\mathrm{T}}(k) \leq v$.

We eventually notice that, once $\dot{m}_{\text {venting }}^{\mathrm{CO}_{2}}(k)$ and $u_{\mathrm{T}}(k)$ have been computed, the original control variables $T_{\mathrm{sa}}(k)$,
$T_{\mathrm{mr}}(k)$ and $\dot{m}_{\mathrm{venting}}(k)$ can be easily computed by simple inversion formulas.

\subsection{Scenario-based Model Predictive Control (MPC)}

As suggested in the modeling section, we decouple the synthesis problem in two separated parts and formulate two problems: the $\mathrm{CO}_{2}$-SMPC problem, which considers model (2), and the T-SMPC problem, which includes model (3).

We also remark that, since the requirements on $\mathrm{CO}_{2}$ concentrations have priority over the thermal comfort ones, the solution computed by the $\mathrm{CO}_{2}$-SMPC is considered by the T-SMPC as a lower bound on the massflow rate.

We thus consider an MPC problem for the control of discrete-time linear systems of the form

$$
\begin{aligned}
x(k+1) & =A x(k)+B(k) u(k)+E w(k) \\
y(k) & =C x(k),
\end{aligned}
$$

where $x(k) \in \mathcal{R}^{n}$ is the state, $u(k) \in \mathcal{R}^{m}$ is the control input, $w(k) \in \mathcal{R}^{r}$ is the stochastic disturbance and $y(k) \in$ $\mathcal{R}^{p}$ is the output. Indeed (6) represents either (2) or (3), depending on the controller under consideration $\left(\mathrm{CO}_{2-}\right.$ SMPC or T-SMPC).

Consider then a prediction horizon $N$ and define

$$
\begin{aligned}
\boldsymbol{x} & :=\left[x(1)^{\mathrm{T}}, \ldots, x(N)^{\mathrm{T}}\right]^{\mathrm{T}}, \\
\boldsymbol{u} & :=\left[u(0)^{\mathrm{T}}, \ldots, u(N-1)^{\mathrm{T}}\right]^{\mathrm{T}}, \\
\boldsymbol{y} & :=\left[y(1)^{\mathrm{T}}, \ldots, y(N)^{\mathrm{T}}\right]^{\mathrm{T}}, \\
\boldsymbol{w} & :=\left[w(0)^{\mathrm{T}}, \ldots, w(N-1)^{\mathrm{T}}\right]^{\mathrm{T}},
\end{aligned}
$$

where $x(k+1)=A x(k)+B u(k)+E w(k)$ denotes the predictions of the state after $k$ time instants into the future. Defining the prediction dynamics matrices $\boldsymbol{A}, \boldsymbol{B}, \boldsymbol{E}$ and $\boldsymbol{C}$ s.t. we can express the output as a function of the initial state $x(0)$ as

$$
\boldsymbol{y}=\boldsymbol{C}_{A} x(0)+\boldsymbol{C}_{B} \boldsymbol{u}+\boldsymbol{C}_{E} \boldsymbol{w} .
$$

The linear constraints on the inputs and outputs over the prediction horizon can instead be generally written as

$$
\begin{aligned}
\boldsymbol{V}_{y} \boldsymbol{y}+\boldsymbol{V}_{u} \boldsymbol{u} & \leq \boldsymbol{v} \\
\boldsymbol{F} \boldsymbol{u} & \leq \boldsymbol{f},
\end{aligned}
$$

where $\boldsymbol{F} \in \mathcal{R}^{q \times m N}, \boldsymbol{f} \in \mathcal{R}^{q}, \boldsymbol{V}_{y} \in \mathcal{R}^{r \times p N}, \boldsymbol{V}_{u} \in \mathcal{R}^{r \times m N}$ and $\boldsymbol{v} \in \mathcal{R}^{r}$.

By replacing (7) in (8), we can write the constraints on the outputs as $\boldsymbol{G}_{u} \boldsymbol{u}+\boldsymbol{G}_{w} \boldsymbol{w} \leq \boldsymbol{g}$, where $\boldsymbol{G}_{u}, \boldsymbol{G}_{w}$ and $\boldsymbol{g}$ are matrices of appropriate dimensions.

MPCs can then be formulated so that it can simultaneously incorporate weather and occupancy forecasts and their uncertainties by means of chance-constrained formulations. It is indeed possible to assume the possibility of violating the comfort bounds on the indoor temperature and $\mathrm{CO}_{2}$ levels with a predefined probability, i.e., formulate output constraints as

$$
\mathbb{P}\left[\boldsymbol{G}_{u} \boldsymbol{u}+\boldsymbol{G}_{w} \boldsymbol{w} \leq \boldsymbol{g}\right] \geq 1-\alpha .
$$

with $\alpha \in[0,1]$ being the violation probability level. In these formulations $\alpha$ represents a tradeoff between performance and constraint satisfaction. 
The cost function represents the energy use over the whole prediction horizon. Denoting by $\boldsymbol{c}^{\mathrm{T}} \boldsymbol{u} \Delta k$ with $\boldsymbol{c} \in \mathbb{R}^{m N}$ the cost vector and $\Delta k$ the sampling period, the control problem can be formally stated as

Problem 1. (Chance Constrained MPC for HVAC Control).

$$
\begin{aligned}
\min _{\boldsymbol{u}} & \boldsymbol{c}^{\mathrm{T}} \boldsymbol{u} \Delta k \\
\text { s.t. } & \mathbb{P}\left[\boldsymbol{G}_{u} \boldsymbol{u}+\boldsymbol{G}_{w} \boldsymbol{w} \leq \boldsymbol{g}\right] \geq 1-\alpha \\
& \boldsymbol{F} \boldsymbol{u} \leq \boldsymbol{f},
\end{aligned}
$$

with $1-\alpha$ the desired probability level for constraint satisfaction.

Chance constrained problems like 1 are generally intractable unless the uncertainties follow specific distributions, e.g., Gaussian or log-concave Kall and Mayer (2005).

However Gaussian assumptions are rather restrictive. To overcome this limitation but still obtain a solvable MPC problem we propose to apply randomized approaches Calafiore (2010), which do not require the specification of particular probability distributions for the uncertainties but only the capability of randomly extracting from them.

The approach is as follows: i) let $\boldsymbol{w}_{1}, \ldots, \boldsymbol{w}_{S}$ be a set of $S$ i.i.d. disturbances samples (called scenarios), $\boldsymbol{w}_{i}:=$ $\left[w_{i}^{\mathrm{T}}(0), \ldots, w_{i}^{\mathrm{T}}(N-1)\right]^{\mathrm{T}}, i=1, \ldots, S$. Then the chance constraints in Problem (1) can be replaced with the deterministic constraints

$$
\boldsymbol{G}_{u} \boldsymbol{u}+\boldsymbol{G}_{w} \boldsymbol{w}_{i}-\boldsymbol{g} \leq \mathbf{0}, \quad i=1, \ldots, S .
$$

Since most of the constraints in (9) are redundant, the only constraint that is required to be satisfied is

$$
\boldsymbol{G}_{u} \boldsymbol{u} \leq \boldsymbol{g}-\max _{i=1, \ldots, S} \boldsymbol{G}_{w} \boldsymbol{w}_{i}
$$

(where the max applies element-wise to $\boldsymbol{G}_{w} \boldsymbol{w}_{i}$ ); ii) soften the constraints in (10) by introducing the slack variables $\epsilon(k) \in \mathbb{R}^{p}$ at each time step $k$, and eventually approximate Problem (1) with

Problem 2. (SMPC for HVAC Control).

$$
\begin{array}{ll}
\min _{\boldsymbol{u}} & \boldsymbol{c}^{\mathrm{T}} \boldsymbol{u} \Delta k+\rho \mathbf{1}^{\mathrm{T}} \boldsymbol{\epsilon} \\
\text { s.t. } & \boldsymbol{G}_{u} \boldsymbol{u} \leq \boldsymbol{g}+\boldsymbol{\epsilon}-\max _{i=1, \ldots S} \boldsymbol{G}_{w} \boldsymbol{w}_{i} \\
& \boldsymbol{F} \boldsymbol{u} \leq \boldsymbol{f}, \\
& \boldsymbol{\epsilon} \geq \mathbf{0},
\end{array}
$$

where $\boldsymbol{\epsilon}$ is the vector containing all the slack variables, $\rho$ is the weight on the slack variables, and $\mathbf{1}$ is a matrix of ones with appropriate dimensions.

We notice two important remarks:

- (how to choose the number of scenarios $S$ ) letting $d=m N$ be the number of decision variables, $S$ can be chosen based on the sufficient condition

$$
S \geq \frac{2}{\alpha}\left(\ln \left(\frac{1}{\beta}\right)+d\right)
$$

that guarantees that considering constraints (9) will lead to a feasible solution for Problem 2 with a confidence level $(1-\beta) \in(0,1)$ with $\beta$ an user-defined parameter (Calafiore, 2010). Experience nonetheless indicates that (11) may be overly pessimistic for an MPC control scheme Zhang et al. (2013).
- (meaning of the slack variables $\epsilon(k)$ 's) the $\epsilon(k)$ 's tune the number of possible constraint violations and guarantee that the problem with sampled constraints is always feasible. If the optimal solution can be obtained without violations of the softened constraints, the slack variables will be set to zero. The designer can thus considerably penalize constraint violations by assigning to the weighting factor a value that is orders of magnitude greater than the other coefficients parameters.

We refer the reader to to Parisio et al. (2013a,b). for details on the generation of the scenarios.

\section{EXPERIMENTAL CASE STUDY}

\subsection{Description of the experimental setup}

We consider a laboratory of approximatively $80 \mathrm{~m}^{2}$ in the ground floor of the Q-building of the KTH Royal Institute of Technology campus in Stockholm. The room has a concrete heavyweight structure with limited glass surface and one external wall, facing South-East, which is partially shaded by a parking lot. As summarized in Figure 1, its HVAC system is composed mainly of two parts: the ventilation system, supplying fresh air, and a radiator heating system.

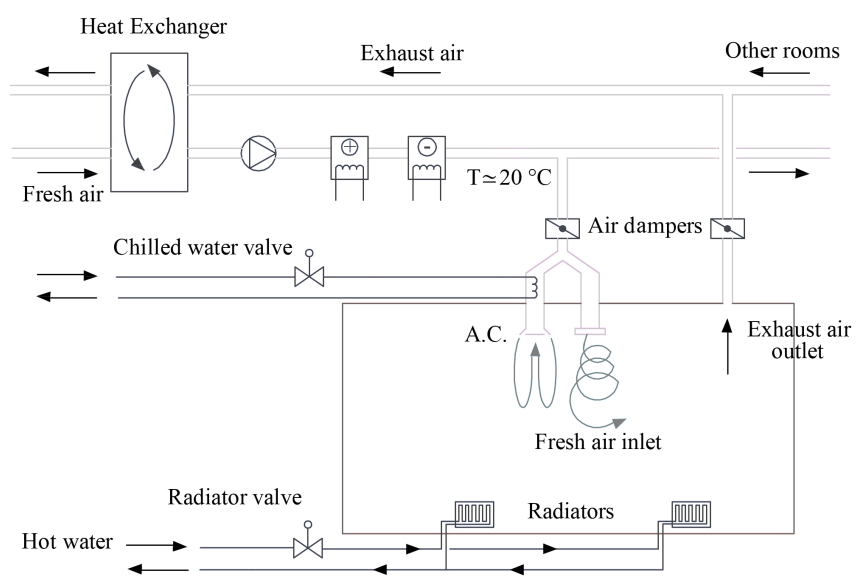

Fig. 1. Scheme of the HVAC system of the testbed.

The air in the ventilation system is pushed from a central fan (not controllable by us) that is active only between 7:00 and 16:00 during working days. Thus no ventilation control action can be carried when this fan is off, and as a consequence we only report tests performed when the central fan was running. A part from this, the ventilation system works as follows: the balanced ventilation system pre-conditions fresh air from outside, distributing it at a temperature of about $20^{\circ} \mathrm{C}$. Part of this generated air flow is then conveyed directly into the room, while part can be further cooled by a cooling coil. Summarizing, the controllable actuators of the ventilation system are 3: two dampers that regulate the opening of the inflow and outflow ducts, and a valve that regulates the temperature of the air chilling circuit. When the central fan is on, a minimum level of the massflow rate is guaranteed in any case. 
The heating system is instead composed by common radiators. The flowing hot water is provided by a district heating, that autonomously decide the temperature of the fluid considering the external temperature conditions. The actuator is the valve regulating the flow of the heating fluid.

Figure 2 depicts the architecture of the implemented control system: the indoor temperature and $\mathrm{CO}_{2}$ concentration are controlled through the ventilation system and radiators, which are actuated using low-level PI controllers. The set-points for the low-level controllers are computed by our SMPC at each time instant, based on new measurements and updated information about weather and occupancy patterns.

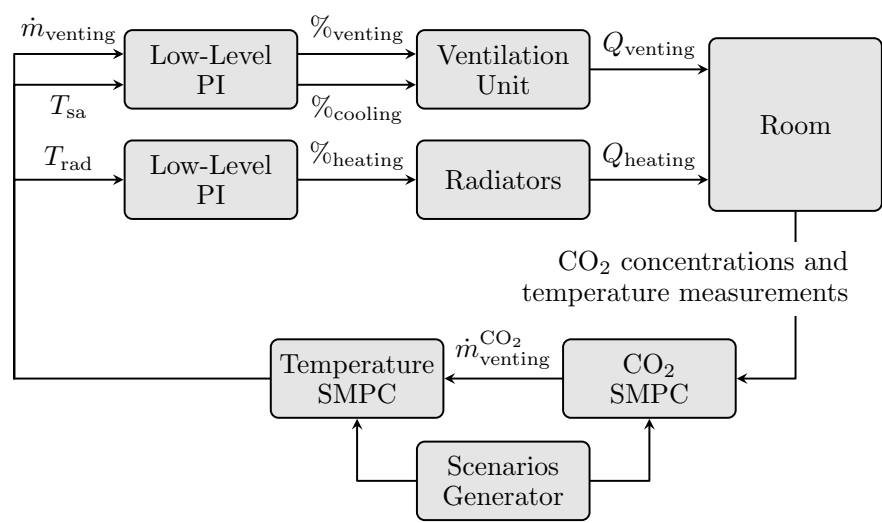

Fig. 2. Architecture of the control system implemented on the testbed.

Denote by $S_{\mathrm{CO}_{2}}$ and $S_{\mathrm{T}}$ the minimum number of scenarios for the $\mathrm{CO}_{2}$-SMPC and the T-SMPC problems respectively, computed according to (11). The scenario-based controller is synthesized according to Algorithm 1.

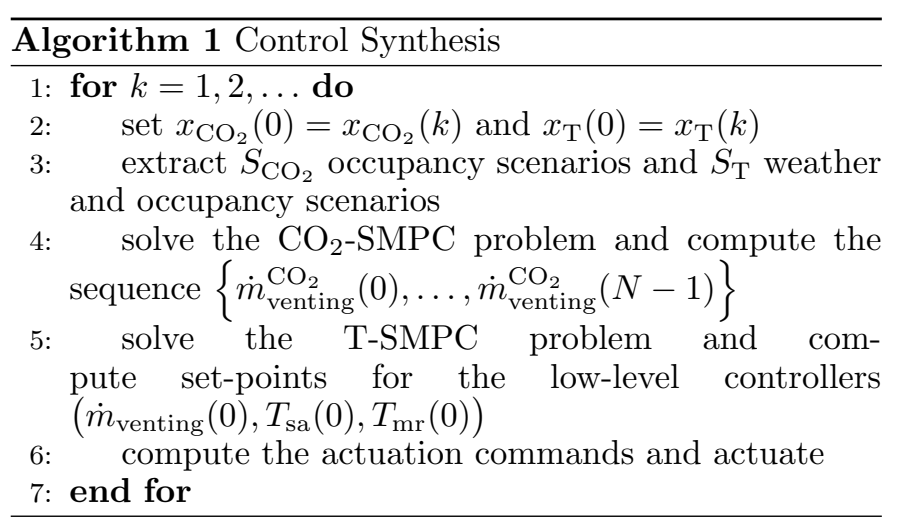

\subsection{Model Validation}

Figures 3 and 4 reports graphical validations of the $\mathrm{CO}_{2}$ and temperature models (2) and (3) against data from the testbed collected during July 2013. We notice that the models accurately capture the dynamics of the systems in consideration, and that they constitute an improvement w.r.t. the models considered in Parisio et al. (2013b).

\subsection{Discussion of Experimental Results}

Our SMPC controller is compared with the current practice, a simple control logic with PI control loops and

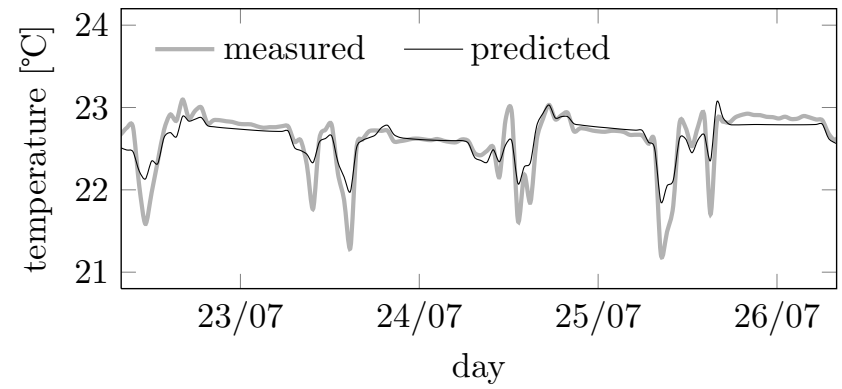

Fig. 3. Validation of the thermal model using the measured temperatures collected from the testbed.

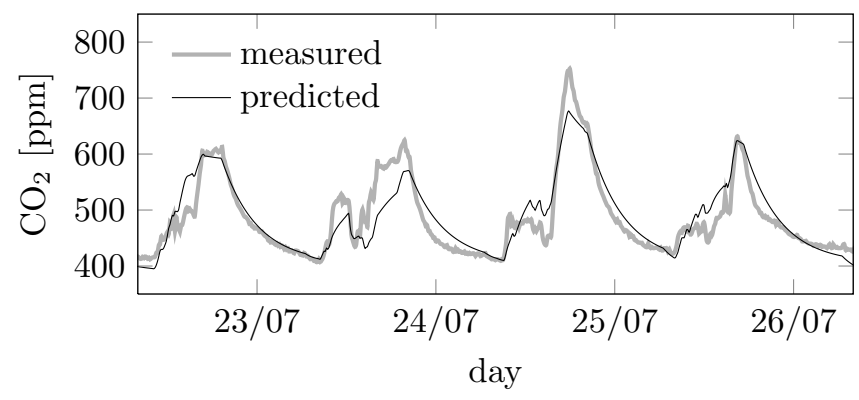

Fig. 4. Validation of the $\mathrm{CO}_{2}$ concentration model using the measured concentrations collected from the testbed.

switching logic, indicated by the acronym "AHC" (from Akademiska Hus, the company managing the building of the testbed).

The sampling time for the SMPC controllers is 10 minutes, while the predictions horizon for the weather, occupancy and solar radiance processes is 8 hours. The comfort range of the indoor temperature is $[20,22]^{\circ} \mathrm{C}$.

Despite the difference in time, the weather conditions during the experiments are similar, as shown in Figure 5, while the occupancy patterns varied during the experimental period. We devise two different occupancy profiles: high and low.

Figure 5 shows experimental results. Each column of Figure 5 refers to one of the tested controllers, while each row depicts the disturbances (i.e., outdoor temperature and occupancy), the control inputs (i.e., supply air temperature and massflow) and the controlled indoor temperature and $\mathrm{CO}_{2}$ levels. The horizontal axis reports the time period of the experiments, from 9:30 to 15:30. We tested the two controllers in different days: November 11 and 13 show high occupancy (thick line) while during November 6 and 21 , a few people where in the testbed room (low occupancy, thin line).

Results for high-occupancy tests. Remarkably, for the SMPC case, the outdoor temperature is lower but its effect on the controllers performance is negligible since the occupancy is the dominating disturbance.

The upper bound on the indoor temperature is violated in all the cases due to the limitations of the ventilation and cooling system. In case of extreme occupancy levels (e.g., 25 people inside the room) and relatively moderately 

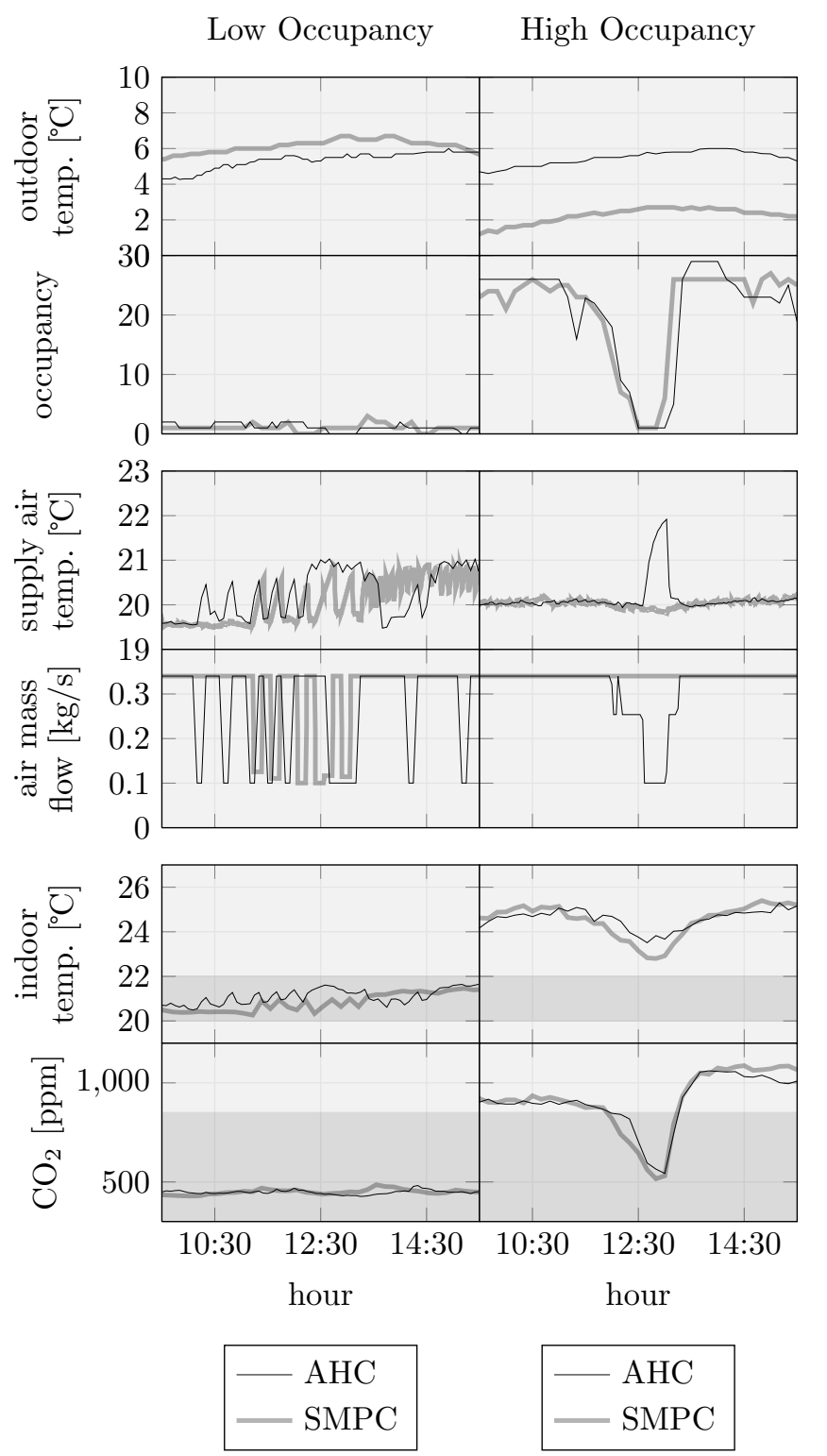

Fig. 5. Disturbances, $\mathrm{CO}_{2}$ levels, indoor temperatures and control inputs profiles for high- and low-occupancy experimental tests. The shaded areas represent the comfort bounds.

high external temperature, full actuation is not sufficient to maintain internal temperature / air $\mathrm{CO}_{2}$ levels within the respective comfort bounds, as shown in some of the investigated experimental cases. This leads to quite similar profiles on the indoor $\mathrm{CO}_{2}$ concentration and temperature for the controllers during the high occupancy hours. Notice that for the AHC controller the supply air temperature exhibits a peak at 13:00 due to the change in the occupancy pattern, while for the SMPC the profile of the supply air temperature from the ventilation system is significantly smoother and does not increase too much. This behavior difference is an example of the added value of the forecasts: the AHC controller does not have knowledge of the upcoming occupancy pattern and decides to turn the ventilation system off at 12:30-13:00, despite the high indoor temperature and the expected number of people.
Results for low-occupancy tests. Considering controller performance in low-occupancy days, we notice that both the disturbances and $\mathrm{CO}_{2}$ profiles are reasonably similar, while the control inputs are different. The supply air temperature for $\mathrm{AHC}$ increases by more than $1{ }^{\circ} \mathrm{C}$ during the time intervals 12:00-13:00 and 14:30-15:30, and the ventilation system is turned on and off often during the morning $(9: 30-12: 30)$. The air supply temperature for SMPC is smoother and kept lower on average. This behavior is mainly caused by a more stressed pre-cooling effect during the morning (the ventilation system is always kept on from 9:30 to roughly 11:30). This leads to an indoor temperature profile with smaller variations, which is a more favorable behavior in terms of comfort. Further, the indoor temperature for the SMPC controller is kept slightly closer to the lower bound.

\section{CONCLUSIONS}

This paper extends the research line started in Parisio et al. $(2013 \mathrm{a}, \mathrm{b})$ by proposing a novel scenario-based model predictive controller for Heating, Ventilation and Air Conditioning (HVAC) systems. The proposed SMPC is able to directly account for the uncertainty of the weather and occupancy forecast in its control decisions.

This paper offers the following major contributions: $i$ ) improvements in the modeling of both the building dynamics and its actuators, leading to a novel and tractable MPC model; ii) improvements in the practical implementation of the proposed control scheme on a real building, which is shown to lead to temperature variations favorably smaller than the ones obtained with the current practice. The easy tunability of the tradeoff between energy usage and comfort violations with one tuning parameter describing the level of constraint violations is a further benefit of our SMPC controller that is to be investigated.

We eventually notice that the proposed SMPC technology is still not completely mature and ready to be massively deployed. Indeed, current implementations require information on the state of the building that up to now are collected using measurement systems usually not present in the majority of the existing buildings (e.g., sensors measuring the temperature of the walls). Thus we devise the necessity of developing advanced estimation schemes that provide indirectly this information. Another important research direction is to extend the control scheme towards networks of thermal zones: the current implementations indeed consider each thermal zone independently and this is inefficient from a optimization problem point of view.

\section{REFERENCES}

Aswani, A., Master, N., and Taneja, J. (2012). Energyefficient building HVAC control using hybrid system LBMPC. In IFAC Conference on Nonlinear Model Predictive Control.

BSI (2008). En 15251:2007: Indoor environmental input parameters for design and assessment of energy performance of buildings addressing indoor air quality, thermal environment, lighting and acoustics. Technical report, British Standards Institute.

Calafiore, G. (2010). Random Convex programs. SIAM Journal on Optimization, 20(6), 3427-3464. 
Chua, K., Chou, S., Yang, W., and Yan, J. (2013). Achieving better energy-efficient air conditioning - A review of technologies and strategies. Applied Energy, 104, 87104.

Costa, A., Keane, M.M., Torrens, J.I., and Corry, E. (2013). Building operation and energy performance: Monitoring, analysis and optimisation toolkit. Applied Energy, 101, 310-316.

European Commission (2008). Debate Europe-building on the experience of plan D for democracy, dialogue and debate. European Economic and Social Committee and the Committee of the Regions, COM 158/4.

Fadzli Haniff, M., Selamat, H., Yusof, R., Buyamin, S., and Sham Ismail, F. (2013). Review of HVAC scheduling techniques for buildings towards energy-efficient and cost-effective operations. Renewable and Sustainable Energy Reviews, 27, 94-103.

Goyal, S., Ingley, H.A., and Barooah, P. (2012). Zone-Level Control Algorithms Based on Occupancy Information for Energy Efficient Buildings. In American Control Conference, 3063-3068.

Gwerder, M. and Toedtli, J. (2005). Predictive control for integrated room automation. In Clima - RHEVA World Congress.

Hua, J. and Karavab, P. (2014). Model Predictive Control Strategies for Buildings with Mixed-Mode Cooling. Building and Environment, 71, 233-244.

Kall, P. and Mayer, J. (2005). Stochastic Linear Programming: Models, Theory, and Computation. SpringerVerlag.

Ma, Y. and Borrelli, F. (2012). Fast stochastic predictive control for building temperature regulation. In American Control Conference, 3075-3080.

Ma, Y., Borrelli, F., and Hencey, B. (2012). Model predictive control for the operation of building cooling systems. IEEE Transactions on Control Systems Technology, 20(3), 796 - 803.

Mady, A.E.d.D., Provan, G.M., Ryan, C., and Brown, K.N. (2011). Stochastic Model Predictive Controller for the Integration of Building Use and Temperature Regulation. In AAAI Conference on Artificial Intelligence, 1371-1376.

Oldewurtel, F., Parisio, A., Jones, C.N., Gyalistras, D., Gwerder, M., Stauch, V., Lehmann, B., and Morari, M. (2012). Use of model predictive control and weather forecasts for energy efficient building climate control. Energy and Buildings, 45(45), 15-27.

Parisio, A., Molinari, M., Varagnolo, D., and Johansson, K.H. (2013a). A scenario-based predictive control approach to Building HVAC managements Systems. In IEEE Conference on Automation Science and Engineering.

Parisio, A., Varagnolo, D., Risberg, D., Pattarello, G., Molinari, M., and Johansson, K.H. (2013b). Randomized Model Predictive Control for HVAC Systems. In Proceedings of the 5th ACM Workshop on Embedded Systems For Energy-Efficient Buildings.

Salsbury, T., Mhaskar, P., and Qin, S.J. (2012). Predictive control methods to improve energy efficiency and reduce demand in buildings. Computers $\& 5$ Chemical Engineering, 51, 77-85.

Sturzenegger, D., Gyalistras, D., Gwerder, M., Sagerschnig, C., Morari, M., and Smith, R.S. (2013). Model
Predictive Control of a Swiss office building. In ClimaRHEVA World Congress.

Treado, S. and Chen, Y. (2013). Saving Building Energy through Advanced Control Strategies. Energies, 6(9), 4769-4785.

Šroký, J., Oldewurtel, F., Cigler, J., and Pr'ivara, S. (2011). Experimental analysis of model predictive control for an energy efficient building heating system. Applied Energy, 88(9), 3079-3087.

Wallace, M., McBride, R., Aumi, S., Mhaskar, P., House, J., and Salsbury, T. (2012). Energy efficient model predictive building temperature control. Chemical Engineering Science, 69(1), 45-58.

Zhang, X., Schildbach, G., Sturzenegger, D., and Morari, M. (2013). Scenario-Based MPC for Energy-Efficient Building Climate Control under Weather and Occupancy Uncertainty. In European Control Conference (ECC), 1029-1034. 